\title{
Spectral filtering approach to the skin blood flow imaging in limbs via infrared thermography: its scope and limits
}

by A.A. Sagaidachnyi, A.V. Fomin, A.V. Skripal, D.A. Usanov

Dept. of Nano - and Biomedical Technology, Saratov State University, Saratov, Russia, andsag@yandex.ru

\begin{abstract}
We develop the new technique for skin blood flow imaging in limbs by means of infrared thermography. In contrast to the lock-in and pulsed phase thermography the proposed method does not use an external heater. Instead, we consider variation of blood volume within the vessels of limbs as a source of thermal waves, travelling through the biotissue. Thus, the blood flow dynamics serves as a non-periodical heater of biological tissue. To reconstruct the blood flow variation from dynamic thermograms we used the spectral filtering approach (SFA), which transforms the temperature signal into the blood flow signal. Reaction of lower limbs on the thermal impact is illustrated by examples of dynamics blood flow maps that are extracted from dynamic thermograms. Limitations and advantages of the blood flow exploration via infrared thermography within each of the frequency range have been discussed.
\end{abstract}

\section{Introduction}

Dynamic IR-thermography has great opportunities in a non-contact monitoring of skin engraftment and researches of burn injuries or chilblains [1-3]. We have established relationships between temperature and skin blood flow oscillations [4]. It turns out that blood volume variation within peripheral vessels can be regarded as a source of thermal waves, travelling through the biotissue.

We applied spectral filtering approach (SFA) to reconstruct the blood flow from skin temperature data in the each pixel of the dynamic thermogram (see section 2.3 for details). Application of SFA to temperature data implies decomposition of the skin temperature signal into spectral components, modification of the spectral components (filtering), followed by composition of the modified spectral components. The resulting video signal can be regarded as dynamics of skin blood flow. The aim of present study is development of Spectral Filtering Approach to the blood flow imaging, and exploration of its scope and limits.

\section{Methods}

\subsection{Data recording}

We used cooled thermal imaging camera ThermaCam SC 3000, Flir Systems as a specimen of modern class equipment for temperature measurements with sensitivity $0.02^{\circ} \mathrm{C}$. Software ThermaCAM Researcher Pro 2.8 SR-2 allowed us to perform measurements in three modes: without software noise reduction (noise reduction= Off), noise reduction $=$ Low, noise reduction=High. All measurements are performed with frame rate $5 \mathrm{fps}$.

\subsection{Permissible frequency range of measurements and sensitivity of thermal imaging}

Analysis of useful and noise components of the temperature signal performed within time and spectral domains in order to estimate the frequency range permissible for established temperature - blood flow relation [5]. The analyzing signals appeared as shown in figure 1. The noise signals recorded in the mode of high (dashed line), low (bold line) and without noise reduction (thin line) (figure $1 \mathrm{a}$ ). Records of temperature oscillations from the distal phalanx of 26 healthy subjects (15 men and 11 women) used to determine the level of useful signal. The subjects were aged 20 to 35 years. Corresponding signals are shown in figure $1 \mathrm{~b}$.

Spectral analysis of the temperature oscillations from finger was carried out to estimate signal-to-noise ratio. Spectral power density calculated by means of squared modulus of Fourier transform (1). Signal-to-noise ratio (SNR) determined by expression (2).

$$
\begin{gathered}
\operatorname{PD}(\mathrm{f})=\left|\frac{1}{t_{\max }} \int_{0}^{\mathrm{t}_{\max }} \mathrm{T}(\mathrm{t}) \cdot \exp (-\mathrm{j} 2 \pi \mathrm{ft}) \mathrm{dt}\right|^{2}, \\
\operatorname{SNR}(\mathrm{f})=10 \cdot \lg \left(\frac{\mathrm{PD}_{\text {signal }}(\mathrm{f})}{\mathrm{PD}_{\text {noise }}(\mathrm{f})}\right),
\end{gathered}
$$

where $t_{\max }$ - the duration of experimental time series, $T(t)$ - the temperature signal, $j$ - the imaginary unit, $t-$ the time, $f$ - the frequency of spectral component, PDsignal and PDnoise- the spectral power densities of useful temperature signal and the noise signal, respectively.

Example of corresponding power density is shown in figure 2. Calculation of temperature signal-to-noise ratio for the group of subjects indicated that cutoff frequency has mean value $0.2 \pm 0.02 \mathrm{~Hz}$. 


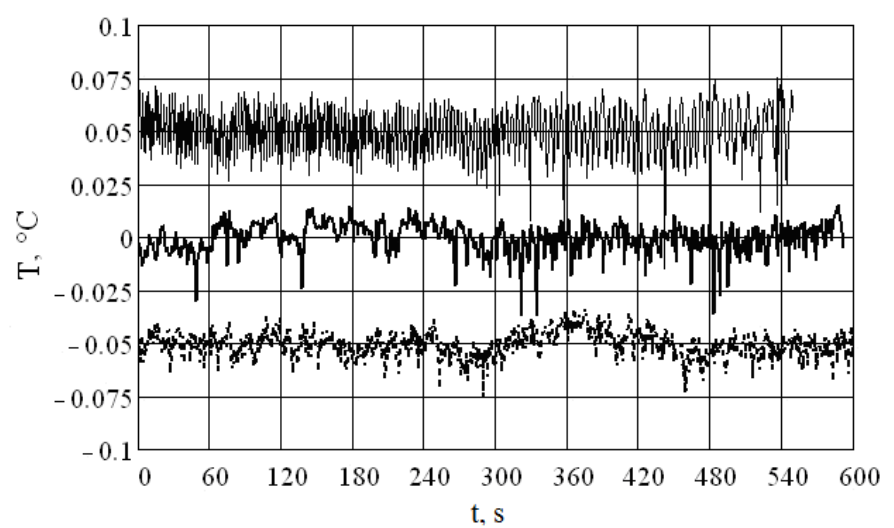

(a)

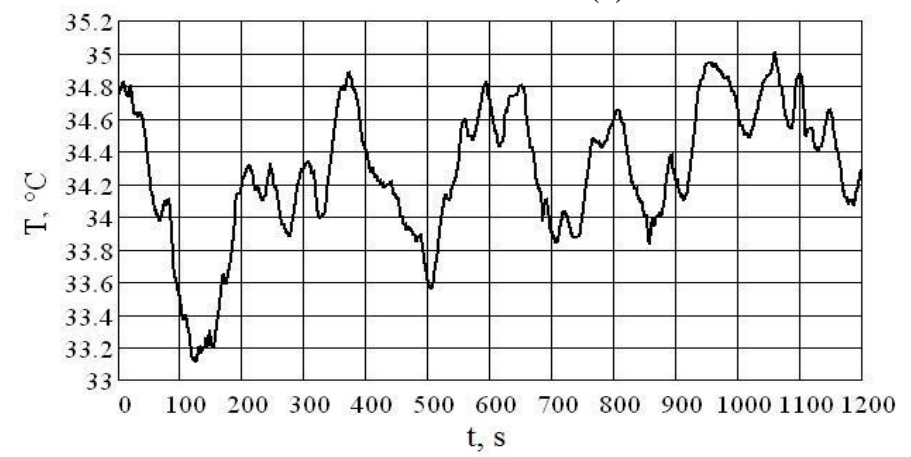

(b)

Fig. 1. Temperature dynamics of the object with constant temperature (pigskin) (a) and the object with oscillating temperature (human hand) under constant environment temperature (b)

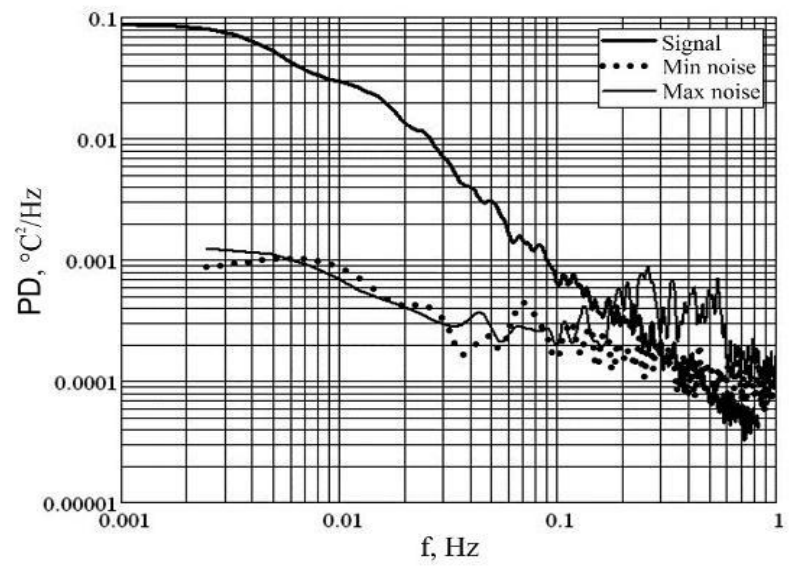

Fig. 2. Spectral analysis of the signal-to-noise ratio for the cooled thermal imaging camera: a-power density of temperature oscillations from finger phalanx (bold line), noise signal without noise reduction (thin line) and with high reduction of the noise (doted-line); $b$ - signal-to-noise ratio of temperature signal for thermal imaging camera without noise reduction

\subsection{Thermography - based blood flow imaging and the spectral filtering approach}

The main feature of the spectral filtering approach as applied to blood flow imaging is that the transformation of temperature signal into blood flow signal occurs in the spectral domain, rather than in the time domain. The general scheme of the method includes decomposition of the temperature signal into spectral components, modification of decomposition coefficients, and finally the composition of modified coefficient which define the curve of blood flow in the time domain. Similarly, the above operations are typical for any case of signal filtering using the amplitude-frequency and phase responses.

The scheme in figure 3 describes steps of the algorithm for thermography-based blood flow imaging. The temperature signal $\mathrm{T}(\mathrm{t})$ (step 1 in Figure 3 ) decomposed into its spectral components (step 2). The phase shift added to decomposition coefficients, and coefficients multiplied by the amplitude coefficient (step 3). Thereafter, performs the inverse transform of modified coefficients, which gives the blood flow oscillations in the time domain (step 4). 


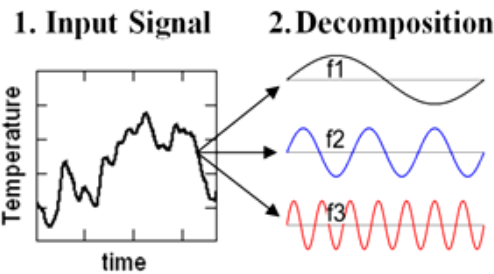

\section{Modification \\ 1. Phase shift \\ 2. Amplitude amplification}

\section{Composition}

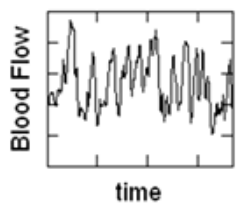

Fig. 3. Scheme of spectral filtering approach applied for converting the skin temperature oscillations into blood flow oscillations

The above algorithm is applied to the each pixel of dynamic thermogram of the object, and then the each value of blood flow put in correspondence with color of selected palette. As a result of processing of temperature data from the surface of an object, the dynamic blood flow maps are formed. In a case of Fourier transform applied for decomposition of the signal, the transformation of the temperature into blood flow can be expressed as (1), and the inverse transformation of blood flow into temperature - as expression (2).

$$
\begin{gathered}
\mathrm{T}(\mathrm{t}) \rightarrow \mathrm{FT}[\mathrm{T}(\mathrm{t})]=\mathrm{S}_{\mathrm{T}}(\mathrm{f}) \rightarrow \exp \left(\mathrm{z} \cdot \sqrt{\frac{\pi \mathrm{f}}{\chi}}(1+\mathrm{j})\right) \cdot \mathrm{S}_{\mathrm{T}}(\mathrm{f})=\widetilde{\mathrm{S}}_{\mathrm{T}}(\mathrm{f}) \rightarrow \operatorname{invFT}\left[\widetilde{\mathrm{S}}_{\mathrm{T}}(\mathrm{f})\right] \rightarrow \mathrm{BF}(\mathrm{t}), \\
\mathrm{BF}(\mathrm{t}) \rightarrow \mathrm{FT}[\mathrm{BF}(\mathrm{t})]=\mathrm{S}_{\mathrm{BF}}(\mathrm{f}) \rightarrow \exp \left(-\mathrm{z} \cdot \sqrt{\frac{\pi \mathrm{f}}{\chi}}(1+\mathrm{j})\right) \cdot \mathrm{S}_{\mathrm{BF}}(\mathrm{f})=\widetilde{\mathrm{S}}_{\mathrm{BF}}(\mathrm{f}) \rightarrow \operatorname{invFT}\left[\widetilde{\mathrm{S}}_{\mathrm{BF}}(\mathrm{f})\right] \rightarrow \mathrm{T}(\mathrm{t}),
\end{gathered}
$$

where FT- the Fourier transform, invFT- the inverse Fourier transform, $\widetilde{\mathrm{S}}_{\mathrm{T}}(\mathrm{f}), \widetilde{\mathrm{S}}_{\mathrm{BF}}(\mathrm{f})$ - the modified Fourier spectra of the temperature and blood flow signals, respectively. Thus, expressions (9) and (10) are reciprocal and gave the opportunity both direct transformation of temperature signal into blood flow and inverse transformation of blood flow signal into temperature.

\section{Results and discussion}

\subsection{Permissible frequency range and temperature sensitivity of thermal imaging}

Figure 4 demonstrates results of estimation of sensitivity required for measurement of skin temperature oscillations provoked by blood flow oscillations within each physiological frequency range. Amplitude of temperature oscillations vs. frequency range on figure 4 can be approximated by the function $a \cdot \exp \left(-b \cdot f^{c}\right)$, where $a=0.8, b=8, c=0.34, f-$ frequency of oscillations (see [5] for details).

The empirical curve in figure 4 can be important to practice. Namely, by means of the curve it is easy to determine the required temperature sensitivity of the camera for the each frequency range of blood flow. The curve, for example, shows that for measurement of temperature oscillations corresponding to respiratory and cardiac ranges it is required sensitivity $\sim 0.01{ }^{\circ} \mathrm{C}$ and $\sim 0.001{ }^{\circ} \mathrm{C}$, respectively. Considering sensitivity equal to $0.02{ }^{\circ} \mathrm{C}$ the curve shows that the upper limit of permissible frequency for my thermal imaging camera is equal to $0.1 \mathrm{~Hz}$. Similar results have been reported previously in $[6,7]$ for the contact skin temperature measurements.

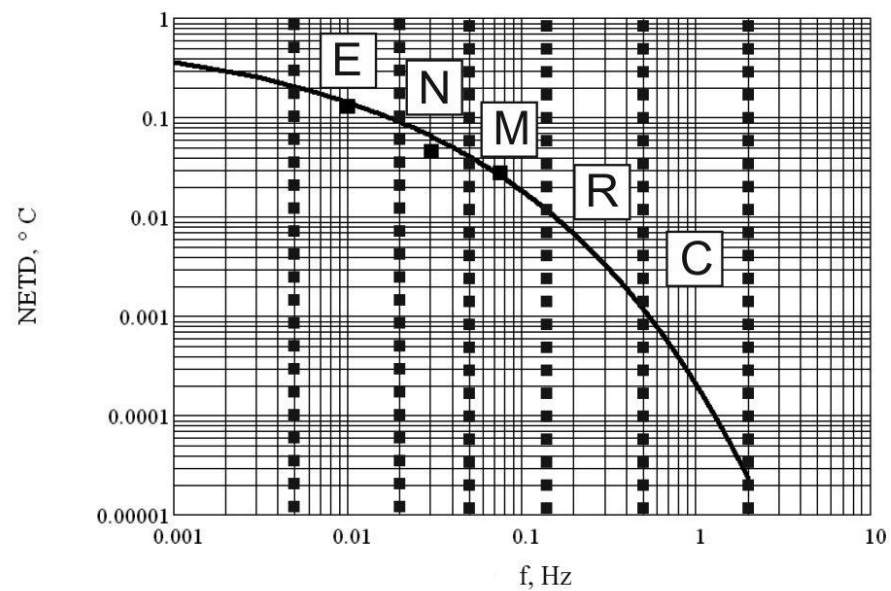

Fig. 4. Dependence of required temperature sensitivity of thermal imaging camera vs. frequency of estimated blood flow oscillations. $E$ - the endothelial, $N$ - the neurogenic, $M$ - the myogenic, $R$ - the respiratory, and $C$ - the cardiac ranges. 


\subsection{Blood flow imaging}

Figure 5 shows an example of blood flow distribution extracted by means of SFA from dynamic thermograms of lower limbs during thermal impact.

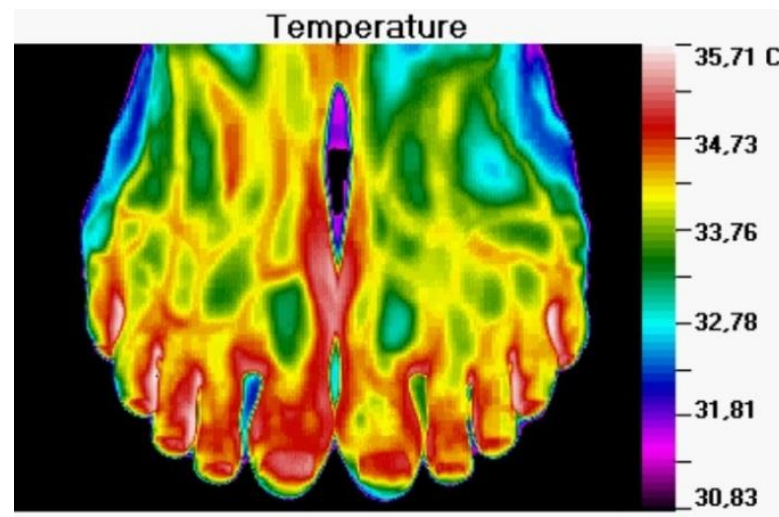

Neurogenic Blood Flow

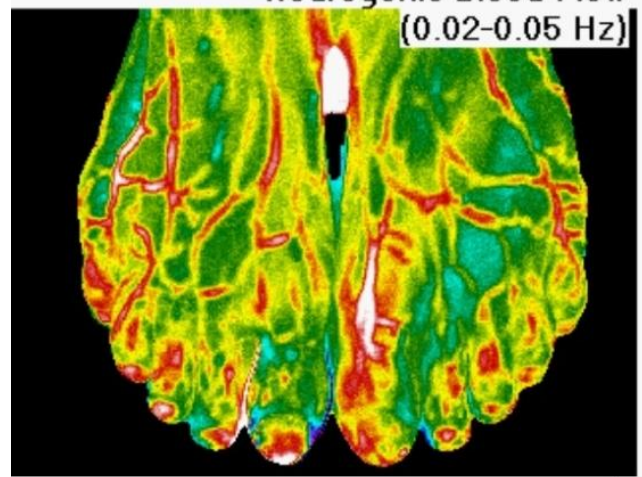

Wide Endothelial Blood Flow

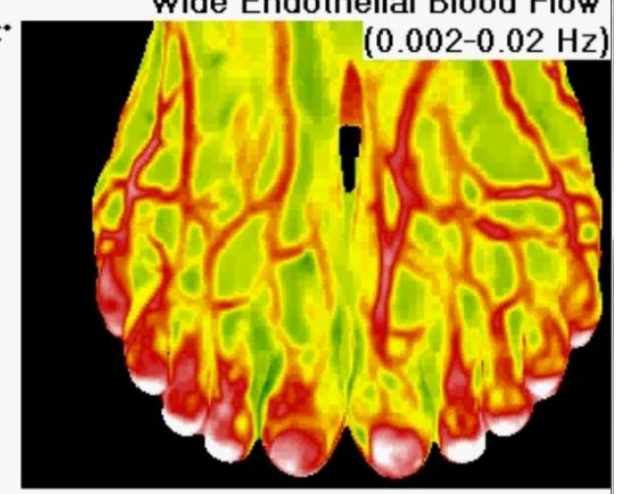

Myogenic Blood Flow

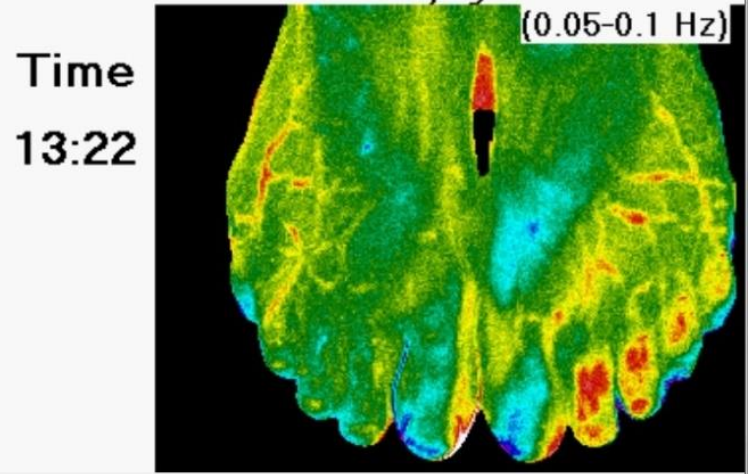

Fig.5. Instantaneous spatial distribution of the blood flow of lower limbs during thermal impact: upper left panel - the temperature, upper right panel - the blood flow within endothelial frequency range, lower left and right panels -blood flow within neurogenic and myogenic frequency ranges, respectively

The figures of blood flow distribution demonstrate increasing of visibility of veins, increasing of toes blood flow within endothelial frequency range and slight contralateral asymmetry of blood flow distribution within neurogenic and myogenic ranges. Different examples of blood flow dynamics extracted from temperature distribution are also available on our web-site LiveTIR.com (http://www.livetir.com/blood-flow-videos.php).

\subsection{Software for converting skin temperature $\leftrightarrow$ blood flow.}

To simplify the process of transformation the temperature signal into the blood flow we have developed multifunctional free accessible program TBF-Converter (see figure 6).

Main features of TBF-Converter are the following:

1. Selection of the direction and method of transformation (Fourier filtering, Shitzer's model).

1.1 Transformation of temperature $\rightarrow$ blood flow signal.

1.2 Transformation of blood flow $\rightarrow$ temperature signal.

2. Fourier filtering of input signals with adjustable frequency range.

4. Definition of properties for a skin, blood and air.

5. Scaling of the curves (normalization or linear scaling).

6. Computation of correlation coefficient between the curves.

TBF-Converter is now available for download on http://livetir.com/software-for-download.php. 


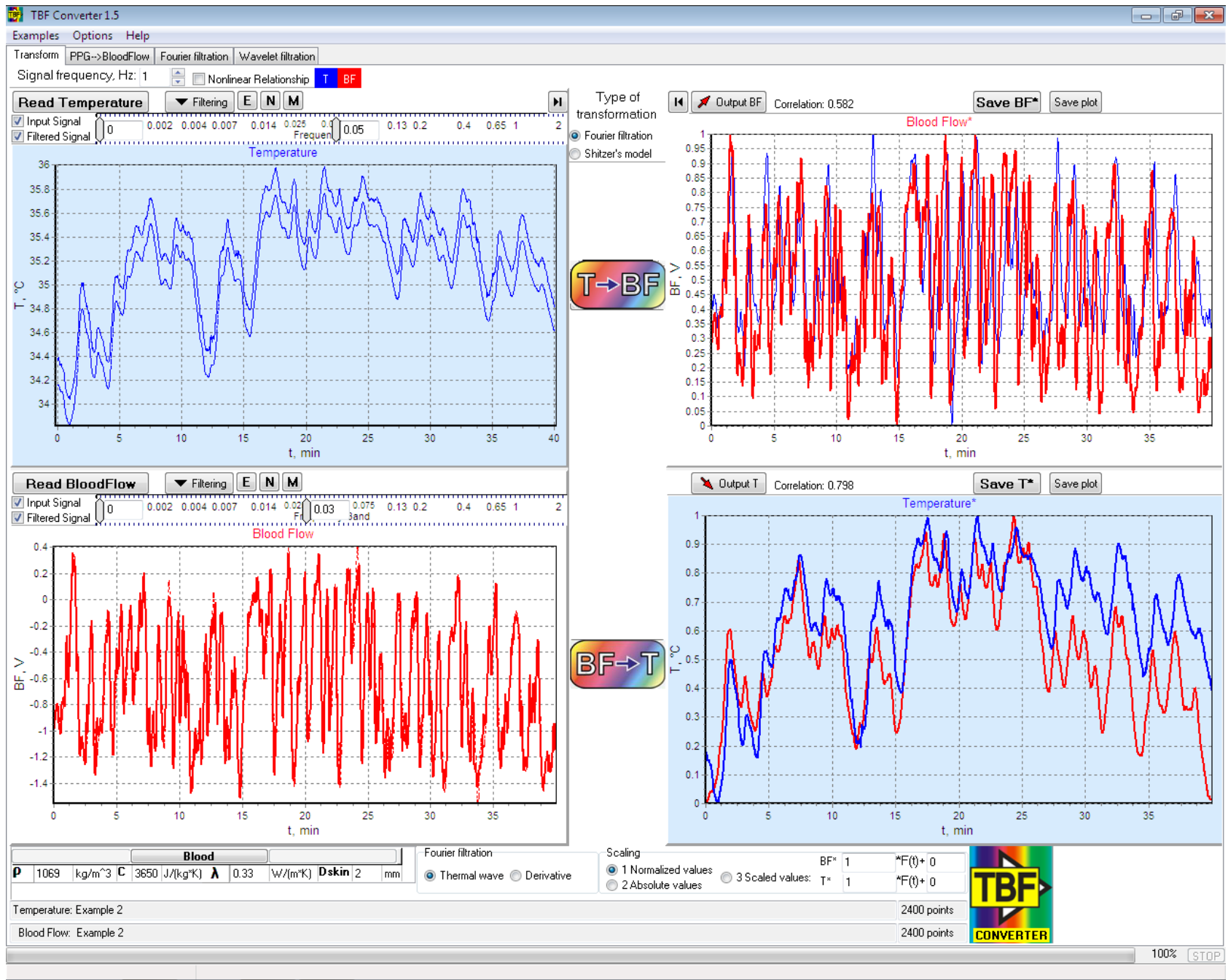

Fig 6. Screenshot of TBF-Converter - software for skin temperature $\leftrightarrow$ blood flow converting

\section{Conclusion}

The described Spectral Filtering Approach to the skin blood flow imaging allows consider thermal imaging as a tool for the evaluation of low-frequency peripheral hemodynamics. The SFA allows transformations the temperature signal into the blood flow and vice versa, and without solving differential equations. The advantages of SFA techniques are as follows: the ability of analyzing of large skin surface area, the blood flow imaging realized with high spatial resolution in the each pixel of the thermogram, the ability to express the intensity of blood flow oscillations in absolute degree measuring units, rather than relative units. Compared to conventional dynamic thermography, mapping of instantaneous blood flow improving the quality of veins images, and allows to reveal the contralateral asymmetry of the blood flow in limbs, which does not appear on the raw thermograms. Results of present study demonstrates limitations when thermal imaging camera using for investigation of temperature oscillations provoked by skin blood flow dynamics. It should be emphasized that modern cooled thermal imaging camera with temperature sensitivity $\sim 0.02^{\circ} \mathrm{C}$ allow to measure of skin temperature oscillations within upper frequency value does not exceed $0.2 \mathrm{~Hz}$. High frequency components of temperature oscillations above $0.2 \mathrm{~Hz}$ have amplitudes comparable with noise amplitudes (figure 2). Estimation of signal-to-noise ration in time domain showed that temperature oscillations exceed noise within endothelial, neurogenic and myogenic frequency ranges below $0.1 \mathrm{~Hz}$. The empirical curve of thermal imaging camera sensitivity as a function of frequency of temperature oscillations (figure 4) gives possibility to evaluate required sensitivity of thermometric equipment. Thus, investigation of skin blood flow oscillations within respiratory and cardiac ranges requires sensitivity of temperature measurements $\sim 0.01$ and $0.001{ }^{\circ} \mathrm{C}$, respectively.

Acknowledgments

The reported study was funded by RFBR according to the research project No. 16-32-00433, by President of the Russian Federation Council for the support of young Russian scientists - PhD, grant № MK-4204.2015.8, and by Ministry of Education and Science of Russian Federation (tasks № 1376 and 1575). 


\section{REFERENCES}

[1] Jiang S.C., Ma N., Li H.J. and Zhang X.X. "Effects of thermal properties and geometrical dimensions on skin burn injuries". Burns, vol. 28, pp. 713-717, 2002.

[2] Liu J., Chen X. and Xu L.X., "New thermal wave aspects on burn evaluation of skin subjected to instantaneous heating". IEEE transactions on biomedical engineering, vol. 46, pp. 420-428, 1999.

[3] Renkielska A., Nowakowski A., Kaczmarek M. and Ruminski J. "Burn depths evaluation based on active dynamic IR thermal imaging - a preliminary study". Burns, vol. 32(7), pp. 867-875, 2006.

[4] Sagaidachnyi A. A., Skripal A. V., Fomin A. V., and Usanov D. A. "Determination of the amplitude and phase relationships between oscillations in skin temperature and photoplethysmography-measured blood flow in fingertips". Physiological measurement, vol. 35(2), 2014.

[5] Sagaidachnyi A. A.; Volkov I. U. and Fomin A. V."Influence of temporal noise on the skin blood flow measurements performed by cooled thermal imaging camera: limit possibilities within each physiological frequency range". Proc. SPIE 9917, Saratov Fall Meeting 2015: Third International Symposium on Optics and Biophotonics and Seventh Finnish-Russian Photonics and Laser Symposium (PALS), 99170N (April 21, 2016), doi:10.1117/12.2229476, http://dx.doi.org/10.1117/12.2229476

[6] Frick P., Mizeva I. and Podtaev S. "Skin temperature variations as a tracer of microvessel tone". Biomedical Signal Processing and Control, vol. 21, pp. 1-7, 2015.

[7] Podtaev S., Morozov M. and Frick P. "Wavelet-based correlations of skin temperature and blood flow oscillations", Cardiovascular Engineering, vol. 8(3), 185-189, 2008. 